\title{
EXPONENTIAL DECAY FOR PRODUCTS OF FOURIER INTEGRAL OPERATORS*
}

\author{
NALINI ANANTHARAMAN ${ }^{\dagger}$
}

\begin{abstract}
This text contains an alternative presentation, and in certain cases an improvement, of the "hyperbolic dispersive estimate" proved in $[1,3]$, where it was used to make progress towards the quantum unique ergodicity conjecture. The main statement gives a sufficient condition to have exponential decay of the norms of long products of sub-unitary Fourier integral operators. The improved version presented here is needed in the two papers [5] and [6].
\end{abstract}

Key words. Semiclassical analysis, Fourier integral operators.

AMS subject classifications. 35S30, 58J40.

1. Introduction. On a Hilbert space $\mathcal{H}$, consider the product $\hat{P}_{n} \hat{P}_{n-1} \cdots \hat{P}_{1}$ of a large number of operators $\hat{P}_{j}$, with $\left\|\hat{P}_{j}\right\|=1$. Think, for instance, of the case where each operator $\hat{P}_{j}$ is an orthogonal projector, or a product of an orthogonal projector and a unitary operator. What kind of geometric considerations can be helpful to prove that the norm $\left\|\hat{P}_{n} \hat{P}_{n-1} \cdots \hat{P}_{1}\right\|$ is strictly less than 1 ? or better, that it decays exponentially fast with $n$ ? In Section 2, we will describe a situation in which $\mathcal{H}=L^{2}\left(\mathbb{R}^{d}\right)$, and the operators $\hat{P}_{j}$ are Fourier integral operators associated to a sequence of canonical transformations $\kappa_{j}$. We will give a "hyperbolicity" condition, on the sequence of transformations $\kappa_{j}$ and on the symbols of the operators $\hat{P}_{j}$, under which we can prove exponential decay of the norms $\left\|\hat{P}_{n} \hat{P}_{n-1} \cdots \hat{P}_{1}\right\|$.

This technique was introduced in $[1,3]$, and is used in $[1,3,4,19,20,6]$ to prove results related to the quantum unique ergodicity conjecture. In $[1,3]$, the proofs are written on a riemannian manifold of negative curvature, for the operators $\hat{P}_{n}=e^{\frac{i \tau h \Delta}{2}} \hat{\chi}_{n}$, in the semiclassical limit $h \longrightarrow 0 ; \triangle$ is the laplacian, $\tau>0$ is fixed, and the operators $\hat{\chi}_{n}$ belong to a finite family of $h$-pseudodifferential operators, microsupported inside compact sets of small diameters. The exponential decay is then used to prove a lower bound on the "entropy" of eigenfunctions, answering by the negative the long-standing question : can a sequence of eigenfunctions concentrate on a closed geodesic, as the eigenvalue goes to infinity ? An expository paper can be found in [16], see also the forthcoming paper [2]. We give here an alternative presentation, based on the use of local adapted symplectic coordinates, which leads in certain cases to an improvement, needed in the two papers [5] and [6].

Let us also mention the work of Nonnenmacher-Zworski [17, 18], Christianson [8, 9, 10], Datchev [11], and Burq-Guillarmou-Hassell [7], who showed how to use these techniques in scattering situations, to prove the existence of a gap below the real axis in the resonance spectrum, and to get local smoothing estimates with loss, as well as Strichartz estimates. In this context, the idea of proving exponential decay for Fourier integral operators was also present, although in an implicit form, in Doi's work $[12]$.

The technique is presented in the first four sections, and the applications needed in $[5,6]$ are stated in section 5 .

\footnotetext{
* Received August 25, 2010; accepted for publication March 22, 2011.

†Laboratoire de Mathématique, Université d'Orsay Paris XI, 91405 Orsay Cedex, France (Nalini. Anantharaman@math.u-psud.fr). The author wishes to acknowledge the support of Agence Nationale de la Recherche, under the grant ANR-09-JCJC-0099-01.
} 
2. A hyperbolic dispersion estimate. In this section, $\mathbb{R}^{d} \times\left(\mathbb{R}^{d}\right)^{*}$ is endowed with the canonical symplectic form $\omega_{o}=\sum_{j=1}^{d} d x_{j} \wedge d \xi_{j}$, where $d x_{j}$ denotes the projection on the $j$-th vector of the canonical basis in $\mathbb{R}^{d}$, and $d \xi_{j}$ is the projection on the $j$-th vector of the dual basis in $\left(\mathbb{R}^{d}\right)^{*}$. The space $\mathbb{R}^{d}$ will also be endowed with its usual scalar product, denoted by $\langle.,$.$\rangle , and we use it to systematically identify \mathbb{R}^{d}$ with $\left(\mathbb{R}^{d}\right)^{*}$.

We consider a sequence of smooth $\left(\mathcal{C}^{\infty}\right)$ transformations $\kappa_{n}: \mathbb{R}^{d} \times \mathbb{R}^{d} \longrightarrow \mathbb{R}^{d} \times \mathbb{R}^{d}$, preserving $\omega_{o}\left(n \in \mathbb{N}^{*}\right)$. We will only be interested in the restriction of $\kappa_{1}$ to a fixed bounded subset of $\mathbb{R}^{d}$, and it is actually sufficient for us to assume that the product $\kappa_{n} \circ \kappa_{n-1} \circ \cdots \circ \kappa_{1}$ is well defined there for all $n$. The Darboux-Lie theorem ensures that every lagrangian foliation can be locally mapped, by a symplectic change of coordinates, to the foliation of $\mathbb{R}^{d} \times \mathbb{R}^{d}$ by the "horizontal" leaves $\mathcal{L}_{\xi_{0}}=\{(x, \xi) \in$ $\left.\mathbb{R}^{d} \times \mathbb{R}^{d}, \xi=\xi_{0}\right\}$. For our purposes (section 5 ), there is no loss of generality if we make the simplifying assumption that each symplectic transformation $\kappa_{n}$ preserves this horizontal foliation. It means that $\kappa_{n}$ is of the form $(x, \xi) \mapsto\left(x^{\prime}, \xi^{\prime}=p_{n}(\xi)\right)$ where $p_{n}: \mathbb{R}^{d} \longrightarrow \mathbb{R}^{d}$ is a smooth function. In more sophisticated words, $\kappa_{n}$ has a generating function of the form

$$
S_{n}\left(x, x^{\prime}, \theta\right)=\left\langle p_{n}(\theta), x^{\prime}\right\rangle-\langle\theta, x\rangle+\alpha_{n}(\theta)
$$

(where $x, x^{\prime}, \theta \in \mathbb{R}^{d}$, and $\alpha_{n}: \mathbb{R}^{d} \longrightarrow \mathbb{R}$ is a smooth function). We have the equivalence

$\left[\left(x^{\prime}, \xi^{\prime}\right)=\kappa_{n}(x, \xi)\right] \Longleftrightarrow\left[\xi=-\partial_{x} S_{n}\left(x, x^{\prime}, \theta\right), \xi^{\prime}=\partial_{x^{\prime}} S_{n}\left(x, x^{\prime}, \theta\right), \partial_{\theta} S_{n}\left(x, x^{\prime}, \theta\right)=0\right]$.

The product $\kappa_{n} \circ \ldots \circ \kappa_{2} \circ \kappa_{1}$ also preserves the horizontal foliation, and it admits the generating function

$$
\begin{array}{r}
\left\langle p_{n} \circ \ldots \circ p_{1}(\theta), x^{\prime}\right\rangle-\langle\theta, x\rangle+\alpha_{1}(\theta)+\alpha_{2}\left(p_{1}(\theta)\right)+\ldots+\alpha_{n}\left(p_{n-1} \circ \ldots \circ p_{1}(\theta)\right) \\
=\left\langle p_{n} \circ \ldots \circ p_{1}(\theta), x^{\prime}\right\rangle-\langle\theta, x\rangle+A_{n}(\theta),
\end{array}
$$

where the equality defines $A_{n}(\theta)$.

If $p$ is a map $\mathbb{R}^{d} \longrightarrow \mathbb{R}^{d}$, we will denote $\nabla p$ the matrix $\left(\frac{\partial p_{i}}{\partial \theta_{j}}\right)_{i j}$, which represents its differential in the canonical basis.

Assumptions (H) : We shall be interested in the following operators, acting on $L^{2}\left(\mathbb{R}^{d}\right)$ :

$$
\hat{P}_{n} f\left(x^{\prime}\right)=\frac{1}{(2 \pi h)^{d}} \int_{x \in \mathbb{R}^{d}, \theta \in \mathbb{R}^{d}} e^{\frac{i S_{n}\left(x, x^{\prime}, \theta\right)}{h}} a^{(n)}\left(x, x^{\prime}, \theta, h\right) f(x) d x d \theta,
$$

where $h>0$ is a "semiclassical" parameter destined to go to 0 . We will assume the following :

(FIO1) For a given $h>0$, the function $\left(x, x^{\prime}, \theta\right) \mapsto a^{(n)}\left(x, x^{\prime}, \theta, h\right)$ is of class $\mathcal{C}^{\infty}$;

(FIO2) When $h \longrightarrow 0$, each $a^{(n)}\left(x, x^{\prime}, \theta, h\right)$ has an asymptotic expansion

$$
a^{(n)}\left(x, x^{\prime}, \theta, h\right) \sim\left(\operatorname{det} \nabla p_{n}(\theta)\right)^{1 / 2} \sum_{k=0}^{\infty} h^{k} a_{k}^{(n)}\left(x, x^{\prime}, \theta\right),
$$

valid up to any order and in all the $\mathcal{C}^{\ell}$ norms on compact sets. 
These conditions imply that the operators $\hat{P}_{n}$ are (semiclassical) Fourier integral operators quantizing the transformations $\kappa_{n}$ [13].

In addition, we assume some uniformity in the behaviour of certain functions as $n$ varies :

(H1) In (FIO2), the asymptotic expansions hold uniformly with respect to $n$ (the derivatives of $a_{k}^{(n)}$ are bounded uniformly in $n$, and the constants in the remainder terms of the expansions are independent of $n$ );

(H2) The functions $p_{n}$ are smooth diffeomorphisms, and all the derivatives of $p_{n}$, of $p_{n}^{-1}$ and of $\alpha_{n}$ are bounded uniformly in $n$.

(H3) For all $n$, for all $(x, \theta),\left(x^{\prime}, \theta^{\prime}\right)$ such that $\left(x^{\prime}, \theta^{\prime}\right)=\kappa_{n}(x, \theta)$, we have $\left|a_{0}^{(n)}\left(x, x^{\prime}, \theta\right)\right| \leq 1$. This condition, together with (H1), ensures that $\left\|\hat{P}_{n}\right\|_{L^{2} \longrightarrow L^{2}} \leq 1+\mathcal{O}(h)$ (uniformly in $n$ ).

Finally, the variables $x, x^{\prime}, \theta$ will be restrained to some fixed bounded subsets of $\mathbb{R}^{d}$, thanks to the following assumption :

(H4) There exist relatively compact subsets $\Omega_{1}, \Omega_{2} \subset \mathbb{R}^{d}$ such that, for all $n$ and $h$, the functions $a^{(n)}\left(x, x^{\prime}, \theta, h\right)$ vanish for $x \notin \Omega_{1}$ or $x^{\prime} \notin \Omega_{1}$ or $\theta \notin \Omega_{2}$.

2.1. Propagation of a single plane wave. The following theorem is essentially proved in [1]. We denote $e_{\xi_{0}, h}$ the function $e_{\xi_{0}, h}(x)=e^{\frac{i\left\langle\xi_{0}, x\right\rangle}{h}}$.

Theorem 2.1. Fix $\xi_{0} \in \mathbb{R}^{d}$. Denote $\xi_{n}=p_{n} \circ \ldots \circ p_{1}\left(\xi_{0}\right)$.

In addition to the assumptions $(H)$ above, assume that

$$
\limsup _{k \longrightarrow+\infty} \frac{1}{k} \log \left\|\nabla\left(p_{n+k} \circ p_{n+k-1} \circ \ldots \circ p_{n+1}\right)\left(\xi_{n}\right)\right\| \leq 0,
$$

uniformly in $n$ (where $\|$.$\| denotes here the norm of a d \times d$ matrix).

Fix $\mathcal{K}, \tilde{\epsilon}>0$ arbitrary, and an integer $M \in \mathbb{N}$ large enough. Then we have, for any $n \leq \mathcal{K}|\log h|$, and $x \in \Omega_{1}$,

$$
\begin{aligned}
& \hat{P}_{n} \circ \ldots \circ \hat{P}_{2} \circ \hat{P}_{1} e_{\xi_{0}, h}(x) \\
& \quad=e^{i \frac{A_{n}\left(\xi_{0}\right)}{h}} e_{\xi_{n}, h}(x)\left(\operatorname{det} \nabla p_{n} \circ \ldots \circ p_{1}\left(\xi_{0}\right)\right)^{1 / 2}\left[\sum_{k=0}^{M-1} h^{k} b_{k}^{(n)}\left(x, \xi_{n}\right)\right]+\mathcal{O}\left(h^{M(1-\tilde{\epsilon})}\right)
\end{aligned}
$$

where the estimate of the remainder holds in the $L^{2}$-norm, as a function of $x$.

The functions $b_{k}^{(n)}$, defined on $\mathbb{R}^{d} \times \mathbb{R}^{d}$, are smooth, supported inside $\Omega_{1} \times \Omega_{2}$, and the function $b_{0}^{(n)}$ is defined by

$$
b_{0}^{(n)}\left(x_{n}, \xi_{n}\right)=\prod_{j=0}^{n-1} a_{0}^{(j)}\left(x_{j}, x_{j+1}, \xi_{j}\right),
$$

where $\xi_{n}=p_{n} \circ \ldots \circ p_{1}\left(\xi_{0}\right)$, and the other terms satisfy $\left(x_{j}, \xi_{j}\right)=\kappa_{j} \circ \ldots \circ \kappa_{1}\left(x_{0}, \xi_{0}\right)$.

The functions $b_{k}^{(n)}$, for $k>0$, have the same support as $b_{0}^{(n)}$. We have $\left|b_{0}^{(n)}\left(x_{n}, \xi_{n}\right)\right| \leq 1$, and besides, we have bounds

$$
\left\|d_{x}^{j} b_{k}^{(n)}\right\|_{\infty} \leq C(k, j, \epsilon) n^{j+3 k} e^{\epsilon(j+2 k) n}
$$

valid for arbitrary $\epsilon>0$, where the prefactor $C(k, j, \epsilon)$ does not depend on $n$.

If we make the assumption that $\left\|\nabla\left(p_{n+k} \circ p_{n+k-1} \circ \ldots \circ p_{n+1}\right)\left(\xi_{n}\right)\right\|$ is bounded above, uniformly in $n, k$, the statement holds with $\epsilon=0$. 
If $n$ is fixed, and if we write $\hat{P}_{n} \circ \ldots \circ \hat{P}_{2} \circ \hat{P}_{1} e_{\xi_{0}, h}(x)$ explicitly as an integral over $\left(\mathbb{R}^{d}\right)^{2 n}$, this theorem is a straightforward application of the stationary phase method. If $n$ is allowed to go to infinity as $h \longrightarrow 0$, our result amounts, in some sense, to applying the method of stationary phase on a space whose dimension goes to $\infty$, and this is known to be very delicate. The theorem was first proved this way, in an unpublished version (available on request or on my webpage) of the paper [1]. A nicer proof, written with the collaboration of Stéphane Nonnenmacher, is available in [1], and has also appeared under different forms in $[3,17]$. In these papers, the proofs are written on a riemannian manifold, for $\hat{P}_{n}=e^{\frac{i \tau \hat{H}}{h}} \hat{\chi}_{n}$, where the operators $\hat{\chi}_{n}$ belong to a finite family of $h$-pseudodifferential operators, microsupported inside compact sets of small diameters, and where $\tau>0$ is fixed and $\hat{H}$ is a semiclassical Schrödinger operator $\left(\hat{H}=-h^{2} \triangle\right.$ in [3]). In local coordinates, and on a manifold of constant negative sectional curvature, the calculations done in $[1,3]$ amount to the simpler statement presented here (see section 5).

In all the papers cited above, the hamiltonian flows under study satisfy a uniform hyperbolicity (or Anosov) property, ensuring a uniform bound $\sup _{\xi \in \Omega_{2}} \| \nabla\left(p_{n} \circ \ldots \circ\right.$ $\left.p_{2} \circ p_{1}\right)(\xi) \| \leq C$, and actually an exponential decay

$$
\sup _{\xi \in \Omega_{2}}\left\|\nabla\left(p_{n} \circ \ldots \circ p_{2} \circ p_{1}\right)(\xi)\right\| \leq C e^{-\lambda n},
$$

with uniform constants $C, \lambda>0$, transversally to some "trivial directions". This is why, following [17], we call our result a hyperbolic dispersion estimate.

2.2. Estimating the norm of $\hat{P}_{n} \circ \ldots \circ \hat{P}_{2} \circ \hat{P}_{1}$. We use the $h$-Fourier transform

$$
\mathcal{F}_{h} u(\xi)=\frac{1}{(2 \pi h)^{d / 2}} \int_{\mathbb{R}^{d}} u(x) e^{-\frac{i\langle\xi, x\rangle}{h}} d x,
$$

the inversion formula

$$
u(x)=\frac{1}{(2 \pi h)^{d / 2}} \int_{\mathbb{R}^{d}} \mathcal{F}_{h} u(\xi) e^{\frac{i\langle\xi, x\rangle}{h}} d \xi
$$

and the Plancherel formula $\|u\|_{L^{2}\left(\mathbb{R}^{d}\right)}=\left\|\mathcal{F}_{h} u\right\|_{L^{2}\left(\mathbb{R}^{d}\right)}$.

We introduce an open relatively compact set $\widetilde{\Omega}_{2} \subset \mathbb{R}^{d}$, that contains $\overline{\Omega_{2}}$. Using the Fourier inversion formula, Theorem 2.1 implies, in a straightforward manner, the following

THEOREM 2.2. In addition to the assumptions (H), we assume that (2.1) holds uniformly in $n$ and $\xi_{0} \in \widetilde{\Omega}_{2}$ (with $\xi_{n}=p_{n} \circ \ldots \circ p_{1}\left(\xi_{0}\right)$ ).

Fix $\mathcal{K}, \epsilon>0$ arbitrary. Then, for $n \leq \mathcal{K}|\log h|$,

$\left\|\hat{P}_{n} \circ \ldots \circ \hat{P}_{2} \circ \hat{P}_{1}\right\|_{L^{2} \longrightarrow L^{2}} \leq \frac{1}{(2 \pi h)^{d / 2}} \sup _{\xi \in \widetilde{\Omega}_{2}}\left|\operatorname{det} \nabla p_{n} \circ \ldots \circ p_{1}(\xi)\right|^{1 / 2}\left(1+\mathcal{O}\left(h n^{3} e^{\epsilon n}\right)\right)$.

Since multiplicative constants do not play a role in the applications we have in mind, all these estimates are to be understood up to a constant.

Of course, since $\left\|\hat{P}_{j}\right\|_{L^{2} \longrightarrow L^{2}} \leq 1+\mathcal{O}(h)$, we always have the trivial bound $\| \hat{P}_{n}$ 。 $\ldots \circ \hat{P}_{2} \circ \hat{P}_{1} \|_{L^{2} \longrightarrow L^{2}} \leq 1+\mathcal{O}(h|\log h|)$. Since we are working in the limit $h \longrightarrow 0$, our estimate can only have an interest if we have an upper bound of the form

$$
\sup _{\xi \in \widetilde{\Omega}_{2}}\left|\operatorname{det} \nabla p_{n} \circ \ldots \circ p_{1}(\xi)\right|^{1 / 2} \leq C e^{-\lambda n}, \quad \lambda>0,
$$


and if $\mathcal{K}$ is large enough. Note that (2.3) is weaker than the condition (2.2).

We now state a refinement of Theorem 2.2. We consider the same family $\hat{P}_{i}$, satisfying assumptions $(\mathrm{H})$. The multiplicative constants in our estimates have no importance, and in what follows we will omit them.

Theorem 2.3. Assume as above that (2.1) holds uniformly in $n$ and $\xi_{0} \in \widetilde{\Omega}_{2}$.

Let $r \leq d$, and assume that the coisotropic foliation by the leaves $\left\{\xi_{r+1}=\right.$ $\left.c_{r+1}, \ldots, \xi_{d}=c_{d}\right\}$ is invariant by each canonical transformation $\kappa_{n}$. In other words, the map $p_{n}$ is of the form

$$
p_{n}\left(\left(\xi_{1}, \ldots, \xi_{r}\right),\left(\xi_{r+1}, \ldots, \xi_{d}\right)\right)=\left(m_{n}\left(\xi_{1}, \ldots, \xi_{d}\right), \tilde{p}_{n}\left(\xi_{r+1}, \ldots, \xi_{d}\right)\right),
$$

where $m_{n}: \mathbb{R}^{d} \longrightarrow \mathbb{R}^{r}$ and $\tilde{p}_{n}: \mathbb{R}^{d-r} \longrightarrow \mathbb{R}^{d-r}$.

Fix $\mathcal{K}, \epsilon>0$ arbitrary. Then there exists $h_{\mathcal{K}}>0$ such that, for any $n \leq \mathcal{K}|\log h|$, and for $h<h_{\mathcal{K}}$,

$$
\begin{aligned}
\left\|\hat{P}_{n} \circ \ldots \circ \hat{P}_{2} \circ \hat{P}_{1}\right\|_{L^{2} \longrightarrow L^{2}} & \\
& \leq \frac{1}{(2 \pi h)^{(r+\epsilon) / 2}} \frac{\sup _{\xi \in \widetilde{\Omega}_{2}}\left|\left(\operatorname{det} \nabla p_{n} \circ \ldots \circ p_{1}(\xi)\right)\right|^{1 / 2}}{\inf _{\xi \in \widetilde{\Omega}_{2}}\left|\left(\operatorname{det} \nabla \tilde{p}_{n} \circ \ldots \circ \tilde{p}_{1}(\xi)\right)\right|^{1 / 2}}\left(1+\mathcal{O}\left(n^{3} h e^{\epsilon n}\right)\right) .
\end{aligned}
$$

In addition, if we make the stronger assumption that $\| \nabla\left(p_{n+k} \circ p_{n+k-1} \circ \ldots \circ\right.$ $\left.p_{n+1}\right)\left(\xi_{n}\right) \|$ is bounded above, uniformly in $n, k$ and for $\xi \in \tilde{\Omega}_{2}$, the statement holds with $\epsilon=0$.

Theorem 2.3 is an improvement of Theorem 2.2 in the case where we have

$$
\frac{1}{(2 \pi h)^{d / 2}} \sup _{\xi \in \Omega_{2}}\left|\left(\operatorname{det} \nabla p_{n} \circ \ldots \circ p_{1}\left(\xi_{0}\right)\right)^{1 / 2}\right| \gg 1
$$

but

$$
\frac{1}{(2 \pi h)^{r / 2}} \frac{\sup _{\xi \in \Omega_{2}}\left|\left(\operatorname{det} \nabla p_{n} \circ \ldots \circ p_{1}(\xi)\right)\right|^{1 / 2}}{\inf _{\xi \in \Omega_{2}}\left|\left(\operatorname{det} \nabla \tilde{p}_{n} \circ \ldots \circ \tilde{p}_{1}(\xi)\right)\right|^{1 / 2}} \ll 1 .
$$

As a trivial example, consider the case where each $\kappa_{n}$ is the identity. Theorem 2.2 gives a non-optimal bound, whereas we can take $r=0$ in Theorem 2.3, and recover the (almost) optimal bound $\left\|\hat{P}_{n} \circ \ldots \circ \hat{P}_{2} \circ \hat{P}_{1}\right\|_{L^{2} \longrightarrow L^{2}} \leq 1+\mathcal{O}\left(h|\log h|^{3}\right)$. A less trivial example will be given in section 5 .

3. Proof of Theorem 2.1. The ideas below are contained in $[1,3]$; however, our notation here is quite different, and we recall (without giving all details) the main steps. In all this section, $M$ is a fixed integer, and all the calculations are done modulo remainders of order $h^{M}$ (with explicit control of the constants).

It is useful to keep in mind the following : if $\left(x^{\prime}, \xi^{\prime}\right)=\kappa_{n} \circ \ldots \circ \kappa_{2} \circ \kappa_{1}(x, \xi)$, we have $\xi^{\prime}=p_{n} \circ \ldots \circ p_{2} \circ p_{1}(\xi)$, and $x=\nabla\left(p_{n} \circ \ldots \circ p_{2} \circ p_{1}\right)^{\top} x^{\prime}+\nabla A_{n}(\xi)$.

3.1. One step of the iteration. Let us first fix $\xi \in \mathbb{R}^{d}$, and look at the action of the operator $\hat{P}_{n}$ on a function of the form

$$
b_{\xi}(x)=e^{\frac{i\langle\xi, x\rangle}{h}} b(x)
$$

where

$$
b(x)=\sum_{k=0}^{M-1} h^{k} b_{k}(x),
$$


and where the functions $b_{k}$ are of class $\mathcal{C}^{\infty}$. Applying $\hat{P}_{n}$ to $b_{\xi}$ will automatically restrict $x$ to a compact subset of $\mathbb{R}^{d}$.

We introduce the following notation :

$$
\left(T_{n}^{\xi} a\right)\left(x^{\prime}\right)=a_{0}^{(n)}\left(x, x^{\prime}, \xi\right) a(x)
$$

where $x$ is the point such that $\left(x^{\prime}, p_{n}(\xi)\right)=\kappa_{n}(x, \xi)$ (in other words, $x=\nabla p_{n}(\xi)^{\top} x^{\prime}+$ $\left.\nabla \alpha_{n}(\xi)\right)$. In the case $a_{0}^{(n)} \equiv 1$, we note that the operator $U_{n}^{\xi}: a \mapsto\left(\operatorname{det} \nabla p_{n}(\xi)\right)^{1 / 2} T_{n}^{\xi} a$ is unitary on $L^{2}\left(\mathbb{R}^{d}\right)$. If we assume (as above) that $\left|a_{0}^{(n)}\left(x, x^{\prime}, \xi\right)\right| \leq 1, U_{n}^{\xi}$ defines a bounded operator on $L^{2}\left(\mathbb{R}^{d}\right)$, of norm $\leq 1$.

A standard application of the stationary phase method yields :

Proposition 3.1.

$$
\hat{P}_{n} b_{\xi}\left(x^{\prime}\right)=e^{i \frac{\alpha_{n}(\xi)+\left\langle p_{n}(\xi), x^{\prime}\right\rangle}{h}}\left(\operatorname{det} \nabla p_{n}(\xi)\right)^{1 / 2}\left[\sum_{k=0}^{M-1} h^{k} b_{k}^{\prime}\left(x^{\prime}\right)\right]+h^{M} R_{M}\left(x^{\prime}\right),
$$

where :

- $b_{0}^{\prime}\left(x^{\prime}\right)=\left(T_{n}^{\xi} b_{0}\right)\left(x^{\prime}\right)$

- $b_{k}^{\prime}\left(x^{\prime}\right)=\sum_{0<l<k-1} D_{n}^{2(k-l)} b_{l}\left(x^{\prime}\right)+\left(T_{n}^{\xi} b_{k}\right)\left(x^{\prime}\right)$, where the operator $D_{n}^{2(k-l)}$ is a differential operator of order $2(k-l)$ (whose expression also depends on $\xi$, although it does not appear in our notation). Its coefficients can be expressed in terms of the derivatives of order $\leq 2(k-l)$ of $a_{l}^{(n)}$, and of order $\leq 2(k-l)+3$ of $p_{n}, p_{n}^{-1}$ and $\alpha_{n}$, at the point $\left(x, x^{\prime}, \xi\right)$, where $\left(x^{\prime}, p_{n}(\xi)\right)=\kappa_{n}(x, \xi)$.

- There exists an integer $N_{d}$ (depending only on the dimension $d$ ), and a positive real number $C$ such that

$$
\left\|R_{M}\right\|_{L^{2}\left(\mathbb{R}^{d}\right)} \leq C \sum_{k=0}^{M-1}\left\|b_{k}\right\|_{\mathcal{C}^{2(M-k)+N_{d}}} .
$$

The constant $C$ can be expressed in terms of a fixed finite number of derivatives of the functions $a_{l}^{(n)}(l \leq M-1), p_{n}, p_{n}^{-1}$ and $\alpha_{n}$ at the point $\left(x, x^{\prime}, \xi\right)$. Under our assumptions (H1) to (H3), $C$ is uniformly bounded for all $n$. Also note that, under (H4), the functions $b_{k}^{\prime}$ are always supported inside the relatively compact set $\Omega_{1}$.

3.2. After many iterations. We now describe the action of the product $\hat{P}_{n} \circ$ $\ldots \circ \hat{P}_{2} \circ \hat{P}_{1}$ on $e_{\xi_{0}, h}$. We will give an approximate expression of $\hat{P}_{n} \circ \ldots \circ \hat{P}_{2} \circ \hat{P}_{1} e_{\xi_{0}, h}(x)$, in the form

$$
e^{i \frac{A_{n}\left(\xi_{0}\right)}{h}} e_{\xi_{n}, h}(x)\left(\operatorname{det} \nabla p_{n} \circ \ldots \circ p_{1}\left(\xi_{0}\right)\right)^{1 / 2}\left[\sum_{k=0}^{M-1} h^{k} b_{k}^{(n)}(x)\right],
$$

as announced in the theorem. This expression will approximate $\hat{P}_{n} \circ \ldots \circ \hat{P}_{2} \circ \hat{P}_{1} e_{\xi_{0}, h}$ up to an error of order $h^{M(1-\tilde{\epsilon})}$ for any $\tilde{\epsilon}>0$. The function $b_{k}^{(n)}(x)$ depends, of course, on $\xi_{0}$, and in the final statement of the theorem we indicated this dependence by writing $b_{k}^{(n)}\left(x, \xi_{n}\right)$ (with $\xi_{n}=p_{n} \circ \ldots \circ p_{1}\left(\xi_{0}\right)$ ).

The method consists in iterating the method described in Section 3.1, controlling carefully how the remainders grow with $n$ in the $L^{2}$-norm. We recall that $\left\|\hat{P}_{n}\right\|_{L^{2}\left(\mathbb{R}^{d}\right)} \leq$ $1+\mathcal{O}(h)$, uniformly in $n$. 
Suppose that, after $n$ iterations, we have proved that

$$
\begin{aligned}
\hat{P}_{n} \circ \ldots \circ \hat{P}_{2} \circ \hat{P}_{1} e_{\xi_{0}, h}(x) & \\
& =e^{i \frac{A_{n}\left(\xi_{0}\right)}{h}} e_{\xi_{n}, h}(x)\left(\operatorname{det} \nabla p_{n} \circ \ldots \circ p_{1}\left(\xi_{0}\right)\right)^{1 / 2}\left[\sum_{k=0}^{M-1} h^{k} b_{k}^{(n)}(x)\right]+h^{M} \mathcal{R}_{M}^{(n)}(x) .
\end{aligned}
$$

We then find a similar expression $\hat{P}_{n+1} \circ \ldots \circ \hat{P}_{2} \circ \hat{P}_{1} e_{\xi_{0}, h}$, with an explicit expression of the family $\left(b_{k}^{(n+1)}\right)_{k=0}^{M-1}$ in terms of $\left(b_{k}^{(n)}\right)_{k=0}^{M-1}$, and a bound on the $L^{2}$-norm of $\mathcal{R}_{M}^{(n+1)}$ in terms of $\left(b_{k}^{(n)}\right)_{k=0}^{M-1}$ and the $L^{2}$-norm of $\mathcal{R}_{M}^{(n)}$.

The calculations done in Section 3.1 allows to describe the action of $\hat{P}_{n+1}$ on $e_{\xi_{n}, h}\left[\sum_{k=0}^{M-1} h^{k} b_{k}^{(n)}\right]:$

$$
\begin{aligned}
& \hat{P}_{n+1} e_{\xi_{n}, h}\left[\sum_{k=0}^{M-1} h^{k} b_{k}^{(n)}\right](x) \\
= & e^{i \frac{\alpha_{n+1}\left(\xi_{n}\right)+\left\langle p_{n+1}\left(\xi_{n}\right), x\right\rangle}{h}}\left(\operatorname{det} \nabla p_{n+1}\left(\xi_{n}\right)\right)^{1 / 2}\left[\sum_{k=0}^{M-1} h^{k} b_{k}^{(n+1)}(x)\right]+h^{M} R_{M}^{(n+1)}(x) .
\end{aligned}
$$

Note that

$$
\prod_{\ell=1}^{n}\left(\operatorname{det} \nabla p_{\ell}\left(\xi_{\ell-1}\right)\right)^{1 / 2}=\left(\operatorname{det} \nabla p_{n} \circ \ldots \circ p_{1}\left(\xi_{0}\right)\right)^{1 / 2}
$$

and

$$
A_{n}\left(\xi_{0}\right)=\alpha_{1}\left(\xi_{0}\right)+\alpha_{2}\left(\xi_{1}\right)+\cdots+\alpha_{n}\left(\xi_{n-1}\right)
$$

so that

$$
\begin{aligned}
& \hat{P}_{n+1} \hat{P}_{n} \circ \ldots \circ \hat{P}_{2} \circ \hat{P}_{1} e_{\xi_{0}, h}(x) \\
= & e^{i \frac{A_{n+1}\left(\xi_{0}\right)}{h}} e_{\xi_{n+1}, h}(x)\left(\operatorname{det} \nabla p_{n+1} \circ \ldots \circ p_{1}\left(\xi_{0}\right)\right)^{1 / 2}\left[\sum_{k=0}^{M-1} h^{k} b_{k}^{(n+1)}(x)\right]+h^{M} \mathcal{R}_{M}^{(n+1)}(x),
\end{aligned}
$$

with the relation $\mathcal{R}_{M}^{(n+1)}=e^{i \frac{A_{n}\left(\xi_{0}\right)}{h}}\left(\operatorname{det} \nabla p_{n} \circ \ldots \circ p_{1}\left(\xi_{0}\right)\right)^{1 / 2} R_{M}^{(n+1)}+\hat{P}_{n+1} \mathcal{R}_{M}^{(n)}$.

We need to control how each term in these expansions will grow with $n$, and in particular, to control the remainder terms. We form an array $B^{(n)}$ that contains all the functions $b_{k}^{(n)}$, and a certain number of higher order differentials :

$$
B_{j, k}^{(n)}=d^{j} b_{k}^{(n)}
$$

with $0 \leq k \leq M-1$ and $0 \leq j \leq 2(M-k)+N_{d}$. The index $k$ indicates the power of $h$, and the index $j$ indicates the number of differentials. Note that $d^{j} b_{k}^{(n)}$ is a (symmetric) covariant tensor field of order $j$ on $\mathbb{R}^{d}$. If $\sigma$ is a covariant tensor field of order $j$ on $\mathbb{R}^{d}$, we define $\|\sigma\|_{\infty}=\sup _{x \in \mathbb{R}^{d}}\left|\sigma_{x}\right|$, where $\left|\sigma_{x}\right|$ is the norm of the $j$-linear form $\sigma_{x}$. By assumption (H4), the forms $d^{j} b_{k}^{(n)}$ all vanish outside the compact set $\Omega_{1}$.

There is a linear relation between $B^{(n)}$ and $B^{(n+1)}$, that we now make a little more explicit. We extend the definition of the operators $T_{n}^{\xi}$ (previously defined on 
functions) to covariant tensor fields, by letting for all $v_{1}, \ldots, v_{j} \in \mathbb{R}^{d}$ (if $\sigma$ is of order j)

$$
\left(T_{n}^{\xi} \sigma\right)_{x^{\prime}}\left(v_{1}, \ldots, v_{j}\right)=a_{0}^{(n)}\left(x, x^{\prime}, \xi\right) \sigma_{x}\left(\nabla p_{n}(\xi)^{\top} v_{1}, \ldots, \nabla p_{n}(\xi)^{\top} v_{j}\right)
$$

where $x=\nabla p_{n}(\xi)^{\top} x^{\prime}$. Taking successive derivatives of the relation

$$
b_{k}^{(n+1)}=\sum_{0 \leq l \leq k-1} D_{n+1}^{2(k-l)} b_{l}^{(n)}+T_{n+1}^{\xi} b_{k}^{(n)}
$$

which appears in Proposition 3.1, we obtain a linear relation of the form :

$$
B^{(n+1)}=K_{n+1} B^{(n)}+L_{n+1} B^{(n)}+T_{n+1}^{\xi} B^{(n)},
$$

where $T_{n+1}^{\xi}$ acts "diagonally", meaning that $\left[T_{n+1}^{\xi} B^{(n)}\right]_{j, k}=T_{n+1}^{\xi}\left(B_{j, k}^{(n)}\right)$. The only information we need about the other terms is that $\left[K_{n+1} B^{(n)}\right]_{j, k}$ depends only on the components $B_{j^{\prime}, l}^{(n)}$, for $l \leq k-1$ and $j^{\prime} \leq 2(k-l)+j$; and $\left[L_{n+1} B^{(n)}\right]_{j, k}$ depends only on the components $B_{j^{\prime}, k}^{(n)}$, with $j^{\prime} \leq j-1$. Besides, we have

$$
\max _{j, k}\left\|\left[K_{n+1} B^{(n)}\right]_{j, k}\right\|_{\infty} \leq C \max _{j, k}\left\|B_{j, k}^{(n)}\right\|_{\infty}
$$

where $C$ does not depend on $n$ by our assumptions (H1) and (H2) - and the same holds with $K_{n+1}$ replaced by $L_{n+1}$.

By induction, we see that $B^{(n)}$ can be expressed as

$$
B^{(n)}=\sum_{M_{\ell} \in\left\{T_{\ell}^{\xi}, K_{\ell}, L_{\ell}\right\}} M_{n} \circ M_{n-1} \circ \cdots \circ M_{1} B^{(0)} .
$$

In a product of the form $M_{n} \circ M_{n-1} \circ \cdots \circ M_{1}$ (where $M_{\ell} \in\left\{T_{\ell}^{\xi}, K_{\ell}, L_{\ell}\right\}$ for all $\ell=1, \ldots, n)$, we see that there can be at most $M$ indices $\ell$ for which $M_{\ell}=K_{\ell}$, and $2 M+N_{d}$ indices $k$ such that $M_{\ell}=L_{\ell}$ (otherwise the product $M_{n} \circ M_{n-1} \circ \cdots \circ M_{1}$ vanishes). Even more precisely, when we write

$$
B_{j, k}^{(n)}=\left[\sum_{M_{\ell} \in\left\{T_{\ell}^{\xi}, K_{\ell}, L_{\ell}\right\}} M_{n} \circ M_{n-1} \circ \cdots \circ M_{1} B^{(0)}\right]_{j, k},
$$

in the right-hand side there can be at most $k$ indices $\ell$ with $M_{\ell}=K_{\ell}$, and $2 k+j$ indices $\ell$ with $M_{\ell}=L_{\ell}$. Hence, the sum has at most $2^{3 k+j}\left(\begin{array}{c}n \\ 3 k+j\end{array}\right) \sim C(k, j) n^{3 k+j}$ terms. Besides, the expression of $B_{j, k}^{(n)}$ involves the action of the operators $T_{\ell}^{\xi}$ on tensor fields of order at most $2 k+j$.

We now use our assumption (2.1). We fix $\epsilon>0$. In the sum (3.3), we use (2.1) to estimate the norm of a string $T_{\ell+m}^{\xi} T_{\ell+m-1}^{\xi} \ldots T_{\ell}^{\xi}$, acting on tensor fields of order $j$ : the norm $\left\|T_{\ell+m}^{\xi} T_{\ell+m-1}^{\xi} \ldots T_{\ell}^{\xi} \sigma\right\|_{\infty}$ can be estimated by $\epsilon^{\epsilon m j}\|\sigma\|_{\infty}$. We obtain

$$
\left\|B_{j, k}^{(n)}\right\|_{\infty} \leq C(k, j, \epsilon) n^{3 k+j} e^{\epsilon n(j+2 k)}
$$

where the term $e^{\epsilon n(j+2 k)}$ comes from our estimate of strings $T_{\ell+m}^{\xi} T_{\ell+m-1}^{\xi} \ldots T_{\ell}^{\xi}$ on tensor fields of order $\leq 2 k+j$, and the fact that the total length of these strings is at 
most $n$. If we make the extra assumption that $\left\|\nabla\left(p_{n+k} \circ p_{n+k-1} \circ \ldots \circ p_{n+1}\right)\left(\xi_{n}\right)\right\|$ is bounded uniformly in $n, k$ and for $\xi \in \tilde{\Omega}_{2}$, the statement holds with $\epsilon=0$.

These estimates (combined with Proposition 3.1) imply that

$$
\left\|R_{M}^{(n+1)}\right\|_{L^{2}\left(\mathbb{R}^{d}\right)} \leq C \sum_{k=0}^{M-1} \sum_{j=0}^{2(M-k)+N_{d}}\left\|B_{j, k}^{(n)}\right\|_{\infty} \leq C(M, \epsilon) n^{3 M+N_{d}} e^{\epsilon n\left(2 M+N_{d}\right)} .
$$

Remember the induction relation

$$
\mathcal{R}_{M}^{(n+1)}=e^{i \frac{A_{n}\left(\xi_{0}\right)}{h}}\left(\operatorname{det} \nabla p_{n} \circ \ldots \circ p_{1}\left(\xi_{0}\right)\right)^{1 / 2} R_{M}^{(n+1)}+\hat{P}_{n+1} \mathcal{R}_{M}^{(n)} .
$$

We have $\left\|\hat{P}_{n+1} \mathcal{R}_{M}^{(n)}\right\|_{L^{2}\left(\mathbb{R}^{d}\right)} \leq(1+\mathcal{O}(h))\left\|\mathcal{R}_{M}^{(n)}\right\|_{L^{2}\left(\mathbb{R}^{d}\right)}$. We thus have an inequality

$$
\left\|\mathcal{R}_{M}^{(n+1)}\right\|_{L^{2}\left(\mathbb{R}^{d}\right)} \leq C(M, \epsilon) n^{3 M+N_{d}} e^{\epsilon n\left(2 M+N_{d}+1\right)}+(1+\mathcal{O}(h))\left\|\mathcal{R}_{M}^{(n)}\right\|_{L^{2}\left(\mathbb{R}^{d}\right)}
$$

with $\left\|\mathcal{R}_{M}^{(0)}\right\|_{L^{2}\left(\mathbb{R}^{d}\right)}=0$. With $\mathcal{O}(h) \leq C h$, this implies that

$$
\left\|\mathcal{R}_{M}^{(n)}\right\|_{L^{2}\left(\mathbb{R}^{d}\right)} \leq C(M, \epsilon) n^{3 M+N_{d}} e^{\epsilon n\left(2 M+N_{d}+1\right)} \frac{(1+C h)^{n}-1}{C h} .
$$

Since we restrict our attention to $n \leq \mathcal{K}|\log h|$, and if we take $M$ larger than $N_{d}$, the right-hand side is bounded by $h^{-\tilde{\epsilon} M}$, where $\tilde{\epsilon}=5 \mathcal{K} \epsilon$.

\section{Proof of Theorems 2.2 and 2.3 .}

4.1. Theorem 2.2. The proof of Theorem 2.2 is now very easy. Let $u \in L^{2}\left(\mathbb{R}^{d}\right)$. We know that

$$
u(x)=\frac{1}{(2 \pi h)^{d / 2}} \int_{\mathbb{R}^{d}} \mathcal{F}_{h} u(\xi) e^{\frac{i\langle\xi, x\rangle}{h}} d \xi .
$$

Let $\widetilde{\Omega}_{2}$ be an open set containing the closure of $\Omega_{2}$. We decompose $u=u_{1}+u_{2}$, where

$$
u_{1}(x)=\frac{1}{(2 \pi h)^{d / 2}} \int_{\widetilde{\Omega}_{2}} \mathcal{F}_{h} u(\xi) e^{\frac{i\langle\xi, x\rangle}{h}} d \xi
$$

and

$$
u_{2}(x)=\frac{1}{(2 \pi h)^{d / 2}} \int_{\mathbb{R}^{d} \backslash \widetilde{\Omega}_{2}} \mathcal{F}_{h} u(\xi) e^{\frac{i\langle\xi, x\rangle}{h}} d \xi
$$

Since $\hat{P}_{1}^{*} \hat{P}_{1}$ is a pseudodifferential operator, which vanishes microlocally outside $\overline{\Omega_{1}} \times$ $\overline{\Omega_{2}}$, we have $\left\|\hat{P}_{1} u_{2}\right\|_{L^{2}\left(\mathbb{R}^{d}\right)}=\mathcal{O}\left(h^{\infty}\right)\left\|u_{2}\right\|_{L^{2}\left(\mathbb{R}^{d}\right)}$.

Concerning $u_{1}$, we apply Theorem 2.1 for each $\xi \in \widetilde{\Omega}_{2}$. We take $n=\mathcal{K}|\log h|$ and choose $M$ accordingly, large enough so that

$$
\mathcal{O}\left(h^{M(1-\tilde{\epsilon})}\right) \ll \sup _{\xi \in \widetilde{\Omega}_{2}}\left|\operatorname{det} \nabla p_{n} \circ \ldots \circ p_{1}(\xi)\right|^{1 / 2} .
$$

This is possible because our assumptions on the derivatives of $p_{n}$ imply a lower bound $\left|\operatorname{det} \nabla p_{n} \circ \ldots \circ p_{1}(\xi)\right| \geq e^{-\beta n}$ for some positive $\beta$.

From Theorem 2.1, we know that

$$
\left\|\hat{P}_{n} \circ \ldots \circ \hat{P}_{2} \circ \hat{P}_{1} e_{\xi, h}\right\|_{L^{2}\left(\mathbb{R}^{d}\right)} \leq\left|\operatorname{det} \nabla p_{n} \circ \ldots \circ p_{1}(\xi)\right|^{1 / 2}\left(1+\mathcal{O}\left(h n^{3} e^{2 \epsilon n}\right)\right)
$$


(for $\epsilon>0$ arbitrary). By a direct application of the triangular inequality, it follows that

$$
\begin{aligned}
& \left\|\hat{P}_{n} \circ \ldots \circ \hat{P}_{2} \circ \hat{P}_{1} u_{1}\right\|_{L^{2}\left(\mathbb{R}^{d}\right)} \\
& \quad \leq \frac{1}{(2 \pi h)^{d / 2}} \sup _{\xi \in \widetilde{\Omega}_{2}}\left|\operatorname{det} \nabla p_{n} \circ \ldots \circ p_{1}(\xi)\right|^{1 / 2}\left(1+\mathcal{O}\left(h n^{3} e^{2 \epsilon n}\right)\right)\left\|\mathcal{F}_{h} u\right\|_{L^{1}\left(\widetilde{\Omega}_{2}\right)} \\
& \leq \frac{1}{(2 \pi h)^{d / 2}} \sup _{\xi \in \widetilde{\Omega}_{2}}\left|\operatorname{det} \nabla p_{n} \circ \ldots \circ p_{1}(\xi)\right|^{1 / 2}\left(1+\mathcal{O}\left(h n^{3} e^{2 \epsilon n}\right)\right)\left|\widetilde{\Omega}_{2}\right|^{1 / 2}\left\|\mathcal{F}_{h} u\right\|_{L^{2}\left(\mathbb{R}^{d}\right)}
\end{aligned}
$$

and our result follows.

\subsection{Theorem 2.3 .}

\subsubsection{The Cotlar-Stein lemma.}

Lemma 4.1. Let $E, F$ be two Hilbert spaces. Let $\left(A_{\alpha}\right) \in \mathcal{L}(E, F)$ be a countable family of bounded linear operators from $E$ to $F$. Assume that for some $R>0$ we have

$$
\sup _{\alpha} \sum_{\beta}\left\|A_{\alpha}^{*} A_{\beta}\right\|^{\frac{1}{2}} \leq R
$$

and

$$
\sup _{\alpha} \sum_{\beta}\left\|A_{\alpha} A_{\beta}^{*}\right\|^{\frac{1}{2}} \leq R
$$

Then $A=\sum_{\alpha} A_{\alpha}$ converges strongly and $A$ is a bounded operator with $\|A\| \leq R$.

The Cotlar-Stein lemma is often used to bound in a precise manner the norm of pseudodifferential operators (see, for example, [13]).

4.2.2. Remember that we assume everywhere that $n=\mathcal{K}|\log h|$, with $\mathcal{K}$ fixed. In order to bound the norm of $\hat{P}_{n} \circ \ldots \circ \hat{P}_{1}$ (modulo $h^{N}$ for arbitrary $N$ ), the results of the previous sections show that it is enough to bound the norm of the operator $A$ defined by

$$
\begin{aligned}
A f\left(x^{\prime}\right)= & \frac{1}{(2 \pi h)^{d}} \int_{\xi \in \widetilde{\Omega}_{2}, x \in \mathbb{R}^{d}}\left(\operatorname{det} \nabla p_{n} \circ \ldots \circ p_{1}(\xi)\right)^{1 / 2} \\
\times & \times\left[\sum_{k=0}^{M-1} h^{k} b_{k}^{(n)}\left(x^{\prime}, \xi_{n}\right)\right] e^{\frac{i}{h}\left(\left\langle\xi_{n}, x^{\prime}\right\rangle+A_{n}(\xi)-\langle\xi, x\rangle\right)} f(x) d x d \xi \\
& =\frac{1}{(2 \pi h)^{d / 2}} \int_{\xi \in \tilde{\Omega}_{2}, x \in \mathbb{R}^{d}}\left(\operatorname{det} \nabla p_{n} \circ \ldots \circ p_{1}(\xi)\right)^{1 / 2} \\
& \times\left[\sum_{k=0}^{M-1} h^{k} b_{k}^{(n)}\left(x^{\prime}, \xi_{n}\right)\right] e^{\frac{i}{h}\left(\left\langle\xi_{n}, x^{\prime}\right\rangle+A_{n}(\xi)\right)} \mathcal{F}_{h} f(\xi) d \xi
\end{aligned}
$$

for a suitable choice of $M$, large. We denote everywhere $\xi_{n}=p_{n} \circ \ldots \circ p_{1}(\xi)$.

We decompose $\mathbb{R}^{d}=\mathbb{R}^{r} \times \mathbb{R}^{d-r}$, and write any $\xi \in \mathbb{R}^{d}$ as $\xi=\left(\xi_{(r)}, \tilde{\xi}\right)$ where $\xi_{(r)} \in$ $\mathbb{R}^{r}$ and $\tilde{\xi} \in \mathbb{R}^{d-r}$. Under our current assumptions, $\xi_{n}$ decomposes as $\xi_{n}=\left(\xi_{n(r)}, \tilde{\xi}_{n}\right)$, where $\tilde{\xi}_{n}=\tilde{p}_{n} \circ \ldots \circ \tilde{p}_{1}(\tilde{\xi})$.

To apply the Cotlar-Stein lemma, we partition the operator $A$ into thin tubes according to the variable $\tilde{\xi}_{n}$; we get a decomposition $A=\sum_{\ell \in \mathbb{Z}^{d-r}} A_{\ell}$, where $A_{\ell}$ and 
$A_{m}$ are microsupported in disjoint tubes if $\|m-\ell\|$ is large. As a result, we show that $\left\|A_{m}^{*} A_{\ell}\right\|$ and $\left\|A_{\ell} A_{m}^{*}\right\|$ are small when $\|m-\ell\|$ is large, and it follows that the norm of $A$ is of the same order as the norms of the individual $A_{\ell}$.

We introduce a (real-valued) smooth compactly supported $\chi$ on $\mathbb{R}^{d-r}$, such that $0 \leq \chi \leq 1$, and having the property that

$$
\sum_{\ell \in \mathbb{Z}^{d-r}} \chi(\tilde{\xi}-\ell)=1
$$

for all $\tilde{\xi} \in \mathbb{R}^{d-r}$. For $h>0, \ell \in \mathbb{Z}^{d-r}$ and $\tilde{\xi} \in \mathbb{R}^{d-r}$, we denote $\chi_{h, \ell}(\tilde{\xi})=\chi\left(\frac{\tilde{\xi}}{2 \pi h}-\ell\right)$. Using the same notation as in (4.1), we define

$$
\begin{aligned}
& A_{\ell} f\left(x^{\prime}\right)=\frac{1}{(2 \pi h)^{d}} \int\left(\operatorname{det} \nabla p_{n} \circ \ldots \circ p_{1}(\xi)\right)^{1 / 2} \\
& \quad \times\left[\sum_{k=0}^{M-1} h^{k} b_{k}^{(n)}\left(x^{\prime}, \xi_{n}\right)\right] \chi_{h, \ell}\left(\tilde{\xi}_{n}\right) e^{\frac{i}{h}\left(\left\langle\xi_{n}, x^{\prime}\right\rangle+A_{n}(\xi)-\langle\xi, x\rangle\right)} f(x) d x d \xi \\
& =\frac{1}{(2 \pi h)^{d / 2}} \int_{\xi \in \tilde{\Omega}_{2}}\left(\operatorname{det} \nabla p_{n} \circ \ldots \circ p_{1}(\xi)\right)^{1 / 2} \\
& \quad \times\left[\sum_{k=0}^{M-1} h^{k} b_{k}^{(n)}\left(x^{\prime}, \xi_{n}\right)\right] \chi_{h, \ell}\left(\tilde{\xi}_{n}\right) e^{\frac{i}{h}\left(\left\langle\xi_{n}, x^{\prime}\right\rangle+A_{n}(\xi)\right)} \mathcal{F}_{h} f(\xi) d \xi
\end{aligned}
$$

It is clear that $A=\sum_{\ell \in \mathbb{Z}^{d-r}} A_{\ell}$. A crucial remark is that the function $\xi \mapsto \chi_{h, \ell}\left(\tilde{\xi}_{n}\right)$, defined on $\Omega_{2}$, is supported in a set of volume $\leq(2 \pi h)^{d-r} \frac{1}{\inf _{\xi \in \tilde{\Omega}_{2}}\left|\left(\operatorname{det} \nabla \tilde{p}_{n} \circ \ldots \circ \tilde{p}_{1}(\xi)\right)\right|}$.

We are going to apply the Cotlar-Stein lemma to this decomposition. Let us write explicitly the expression for the adjoint :

$$
\begin{aligned}
A_{\ell}^{*} f(x)= & \frac{1}{(2 \pi h)^{d}} \int\left(\operatorname{det} \nabla p_{n} \circ \ldots \circ p_{1}(\xi)\right)^{1 / 2} \\
& \times\left[\sum_{k=0}^{M-1} h^{k} \overline{b_{k}^{(n)}}\left(x^{\prime}, \xi_{n}\right)\right] \chi_{h, \ell}\left(\tilde{\xi}_{n}\right) e^{-\frac{i}{h}\left(\left\langle\xi_{n}, x^{\prime}\right\rangle+A_{n}(\xi)-\langle\xi, x\rangle\right)} f\left(x^{\prime}\right) d x^{\prime} d \xi
\end{aligned}
$$

We shall evaluate the norm of $A_{m}^{*} A_{\ell}$ and $A_{\ell} A_{m}^{*}$, for all $m, \ell \in \mathbb{Z}^{d-r}$.

4.2.3. Norm of $A_{m}^{*} A_{\ell}$. We evaluate the norm of $A_{m}^{*} A_{\ell}$ acting on $L^{2}\left(\mathbb{R}^{d}\right)$ by studying the scalar product $\left\langle A_{\ell} f, A_{m} f\right\rangle$ for $f \in L^{2}\left(\mathbb{R}^{d}\right)$. Using expression (4.2) and bilinearity of the scalar product, we will bound the scalar product $\left\langle A_{\ell} f, A_{m} f\right\rangle$ by studying separately each bracket

$$
\chi_{h, \ell}\left(\tilde{\xi}_{n}\right) \chi_{h, m}\left(\tilde{\xi}_{n}^{\prime}\right)\left\langle\left[\sum_{k=0}^{M-1} h^{k} b_{k}^{(n)}\left(x^{\prime}, \xi_{n}\right)\right] e^{\frac{i}{h}\left\langle\xi_{n}, x^{\prime}\right\rangle},\left[\sum_{k=0}^{M-1} h^{k} b_{k}^{(n)}\left(x^{\prime}, \xi_{n}^{\prime}\right)\right] e^{\frac{i}{h}\left\langle\xi_{n}^{\prime}, x^{\prime}\right\rangle}\right\rangle_{L_{x^{\prime}}^{2}} .
$$

Using the notation of $\S 4.2 .2$, we decompose the complex phase $\left\langle\xi_{n}, x^{\prime}\right\rangle-\left\langle\xi_{n}^{\prime}, x^{\prime}\right\rangle$ into $\left\langle\xi_{n(r)}, x_{(r)}^{\prime}\right\rangle-\left\langle\xi_{n(r)}^{\prime}, x_{(r)}^{\prime}\right\rangle+\left\langle\tilde{\xi}_{n}, \tilde{x}^{\prime}\right\rangle-\left\langle\tilde{\xi}_{n}^{\prime}, \tilde{x}^{\prime}\right\rangle$. In the integral defining the scalar product (4.4), we perform an integration by parts with respect to $\tilde{x}^{\prime} \in \mathbb{R}^{d-r}$ : we integrate $N$ times the function $e^{\frac{i}{h}\left\langle\tilde{\xi}_{n}, \tilde{x}^{\prime}\right\rangle-\left\langle\tilde{\xi}_{n}^{\prime}, \tilde{x}^{\prime}\right\rangle}$ and differentiate the functions $b_{k}^{(n)}\left(x^{\prime}, \xi_{n}\right)$. Using the estimates of Theorem 2.1, we obtain 
Proposition 4.2.

$$
\begin{aligned}
\chi_{h, \ell}\left(\tilde{\xi}_{n}\right) \chi_{h, m}\left(\tilde{\xi}_{n}^{\prime}\right) \mid\left\langle\left[\sum_{k=0}^{M-1} h^{k} b_{k}^{(n)}\left(x^{\prime}, \xi_{n}\right)\right] e^{\frac{i}{h}\left\langle\xi_{n}, x^{\prime}\right\rangle},\right. & {\left.\left[\sum_{k=0}^{M-1} h^{k} b_{k}^{(n)}\left(x^{\prime}, \xi_{n}^{\prime}\right)\right] e^{\frac{i}{h}\left\langle\xi_{n}^{\prime}, x^{\prime}\right\rangle}\right\rangle \mid } \\
& \leq C(\epsilon) n^{N} e^{\epsilon N n} \frac{1}{(\|m-\ell\|+1)^{N}}
\end{aligned}
$$

for $\epsilon>0$ arbitrary.

The integer $N$ will be chosen soon, and only depends on the dimension $d$.

REMARK 4.3. The factor $e^{\epsilon N n}$ comes from the estimate of the derivatives of order $N$ with respect to $x^{\prime}$ of the functions $b_{k}^{(n)}\left(x^{\prime}, \xi_{n}\right)$, given in Theorem 2.1. If we make the assumption that $\left\|\nabla\left(p_{n+k} \circ p_{n+k-1} \circ \ldots \circ p_{n+1}\right)\left(\xi_{n}\right)\right\|$ is bounded above, the statement holds with $\epsilon=0$.

We now use the bilinearity of the scalar product, and the fact that

$$
\begin{aligned}
& \left\|\chi_{h, \ell}\left(\tilde{\xi}_{n}\right) \mathcal{F}_{h} f(\xi)\right\|_{L^{1}\left(\widetilde{\Omega}_{2}\right)} \\
& \quad \leq(2 \pi h)^{(d-r) / 2} \frac{1}{\inf _{\xi \in \widetilde{\Omega}_{2}}\left|\left(\operatorname{det} \nabla \tilde{p}_{n} \circ \ldots \circ \tilde{p}_{1}(\xi)\right)\right|^{1 / 2}}\left\|\mathcal{F}_{h} f(\xi)\right\|_{L^{2}\left(\mathbb{R}^{d}\right)} .
\end{aligned}
$$

Combined with expression (4.2), this yields that

$$
\left\|A_{m}^{*} A_{\ell}\right\| \leq C(\epsilon) e^{\epsilon N n} \frac{1}{(\|m-\ell\|+1)^{N}} \frac{1}{(2 \pi h)^{r}} \frac{\sup _{\xi \in \tilde{\Omega}_{2}}\left|\left(\operatorname{det} \nabla p_{n} \circ \ldots \circ p_{1}(\xi)\right)\right|}{\inf _{\xi \in \widetilde{\Omega}_{2}}\left|\left(\operatorname{det} \nabla \tilde{p}_{n} \circ \ldots \circ \tilde{p}_{1}(\xi)\right)\right|}
$$

Looking at the statement of the Cotlar-Stein lemma, we see that we must choose $N$ large enough such that $\sum_{\ell \in \mathbb{Z}^{d-r}} \frac{1}{(\|\ell\|+1)^{N / 2}}<+\infty$.

4.2.4. Norm of $A_{\ell} A_{m}^{*}$. This step is actually shorter than the previous one. We now have to evaluate the scalar product $\left\langle A_{\ell}^{*} f, A_{m}^{*} f\right\rangle$ for $f \in L^{2}\left(\mathbb{R}^{d}\right)$, and we use the expression (4.3) of the adjoint. We do not need integration by parts, as we see directly that $\left\langle A_{\ell}^{*} f, A_{m}^{*} f\right\rangle$ vanishes as soon as $\|m-\ell\|$ is too large (in fact, the supports of $\chi_{h, \ell}$ and $\chi_{h, m}$ are disjoint if $\|m-\ell\|>C$, where $C$ is fixed and depends only on the support of $\chi$ ). In what follows we consider the case $\|m-\ell\| \leq C$. We see that $A_{\ell}^{*} f$ is the $\mathcal{F}_{h}$-transform of

$$
\begin{aligned}
F_{\ell}: \xi \mapsto \frac{1}{(2 \pi h)^{d / 2}} \int\left(\operatorname{det} \nabla p_{n} \circ \ldots \circ p_{1}(\xi)\right)^{1 / 2} \\
\times\left[\sum_{k=0}^{M-1} h^{k} \overline{b_{k}^{(n)}}\left(x^{\prime}, \xi_{n}\right)\right] \chi_{h, \ell}\left(\tilde{\xi}_{n}\right) e^{-\frac{i}{h}\left(\left\langle\xi_{n}, x^{\prime}\right\rangle+A_{n}(\xi)\right)} f\left(x^{\prime}\right) d x^{\prime} .
\end{aligned}
$$

We recall that each $b_{k}^{(n)}\left(x^{\prime}, \xi_{n}\right)$ is supported in $\left\{x^{\prime} \in \Omega_{1}\right\}$, and we bound

$$
\begin{aligned}
& \left\|F_{\ell}\right\|_{L^{2}\left(\mathbb{R}^{d}\right)} \leq \frac{1}{(2 \pi h)^{d / 2}} \sup _{\xi \in \widetilde{\Omega}_{2}}\left|\left(\operatorname{det} \nabla p_{n} \circ \ldots \circ p_{1}(\xi)\right)\right|^{1 / 2}(2 \pi h)^{(d-r) / 2} \\
& \times \frac{1}{\inf _{\xi \in \tilde{\Omega}_{2}}\left|\left(\operatorname{det} \nabla \tilde{p}_{n} \circ \ldots \circ \tilde{p}_{1}(\xi)\right)\right|^{1 / 2}}\|f\|_{L^{1}\left(\Omega_{1}\right)},
\end{aligned}
$$


and $\|f\|_{L^{1}\left(\Omega_{1}\right)} \leq\left|\Omega_{1}\right|^{1 / 2}\|f\|_{L^{2}\left(\mathbb{R}^{d}\right)}$. We obtain the bound

$$
\left\|A_{\ell} A_{m}^{*}\right\| \leq \frac{1}{(2 \pi h)^{r}} \frac{\sup _{\xi \in \widetilde{\Omega}_{2}}\left|\left(\operatorname{det} \nabla p_{n} \circ \ldots \circ p_{1}(\xi)\right)\right|}{\inf _{\xi \in \widetilde{\Omega}_{2}}\left|\left(\operatorname{det} \nabla \tilde{p}_{n} \circ \ldots \circ \tilde{p}_{1}(\xi)\right)\right|},
$$

and $\left\|A_{\ell} A_{m}^{*}\right\|=0$ if $\|\ell-m\|>C$. Estimates (4.6) and (4.7), combined with the Cotlar-Stein lemma, yield Theorem 2.3. The last statement of the theorem comes from Remark 4.3.

5. Examples. We now give an application of Theorems 2.2 and 2.3. These results are needed in [6] and [5].

Let $\mathbf{Y}$ be a $d$-dimensional $\mathcal{C}^{\infty}$ manifold. The cotangent bundle $T^{*} \mathbf{Y}$ is endowed with its canonical symplectic form, denoted by $\omega$. Let $H: T^{*} \mathbf{Y} \longrightarrow \mathbb{R}$ be a smooth function (hamiltonian), and let $\Phi_{H}^{t}: T^{*} \mathbf{Y} \longrightarrow T^{*} \mathbf{Y}$ be the corresponding hamiltonian flow (we assume for simplicity that $\left(\Phi_{H}^{t}\right)$ is complete).

We assume that we have a smooth foliation $\mathcal{F}$ of $T^{*} \mathbf{Y}$ by lagrangian leaves (in the sequel we shall simply speak about a "lagrangian foliation"), such that $\mathcal{F}$ is $\Phi_{H^{-}}^{t}$ invariant : $\Phi_{H}^{t}(\mathcal{F})=\mathcal{F}$ for all $t$. Let $O \subset T^{*} \mathbf{Y}$ be an open, relatively compact subset of $T^{*} \mathbf{Y}$; we assume that we have a finite open covering of $O, O \subset O_{1} \cup O_{2} \cup \ldots \cup O_{K}$, and for all $k=1, \ldots, K$, a smooth symplectic coordinate chart $\Psi_{k}:\left(O_{k}, \omega\right) \longrightarrow\left(\mathbb{R}^{2 d}, \omega_{o}\right)$ which maps $O_{k}$ to a ball in $\mathbb{R}^{2 d}$, and the foliation $\left.\mathcal{F}\right\rceil_{O_{k}}$ to the horizontal foliation of that ball.

We now describe the operators $\hat{P}_{k}$ to which we shall apply the main results. Let $\hat{H}$ be a self-adjoint $h$-pseudodifferential operator with principal symbol $H$. We fix a family $\hat{\chi}_{1}, \ldots, \hat{\chi}_{K}$ of $h$-pseudodifferential operators, microsupported inside $O_{k}$. We also assume that its principal symbol $\chi_{k}$ (which is a smooth function on $T^{*} \mathbf{Y}$ ) satisfies $\left\|\chi_{k}\right\|_{\mathcal{C}^{0}} \leq 1$. Fix, finally, a time step $\tau>0$ and a sequence $\left(\alpha_{0}, \alpha_{2}, \ldots, \alpha_{n-1}\right) \in$ $\{1, \ldots, K\}^{n}$. We shall use Theorems 2.2 and 2.3 to estimate the norm of the product $\prod_{k=0}^{n-1} \hat{\chi}_{\alpha_{k+1}} e^{-\frac{i \tau \hat{H}}{h}} \hat{\chi}_{\alpha_{k}}$. The operator $\hat{P}_{k}$ will be $\hat{\chi}_{\alpha_{k+1}} e^{-\frac{i \tau \hat{H}}{h}} \hat{\chi}_{\alpha_{k}}$, read in an adapted coordinate system.

We fix a collection of Fourier integral operators $U_{k}: L^{2}(\mathbf{Y}) \longrightarrow L^{2}\left(\mathbb{R}^{d}\right)$, quantizing the canonical transformation $\Psi_{k}(k=0, \ldots n-1)$, and such that the pseudodifferential operator $U_{k}^{*} U_{k}$ satisfies $U_{k}^{*} U_{k} \hat{\chi}_{k}=\hat{\chi}_{k}+\mathcal{O}\left(h^{\infty}\right)$ and $\hat{\chi}_{k}=\hat{\chi}_{k} U_{k}^{*} U_{k}+\mathcal{O}\left(h^{\infty}\right)$ (where the $\mathcal{O}$ is to be understood in the $L^{2}(\mathbf{Y})$-operator norm). We take $\hat{P}_{k}=$ $U_{\alpha_{k+1}} \hat{\chi}_{\alpha_{k+1}} e^{-\frac{i \tau \hat{H}}{h}} \hat{\chi}_{\alpha_{k}} U_{\alpha_{k}}^{*}$. It is a Fourier integral operator $L^{2}\left(\mathbb{R}^{d}\right) \longrightarrow L^{2}\left(\mathbb{R}^{d}\right)$, associated with the canonical transformation $\kappa_{k}=\Psi_{\alpha_{k+1}} \Phi_{H}^{\tau} \Psi_{\alpha_{k}}^{-1}$, which by construction preserves the horizontal foliation. These operators satisfy all assumptions $(\mathrm{H})$, hence we can apply to them Theorems 2.2 and 2.3. Actually, we may allow the collection of coordinate charts $\left(\Psi_{\alpha_{0}}, \ldots, \Psi_{\alpha_{n-1}}\right)$ to depend on the whole sequence $\left(\alpha_{0}, \alpha_{1}, \ldots, \alpha_{n-1}\right)$, the important requirement being that the derivatives of $\Psi_{\alpha_{k}}$ and $\Psi_{\alpha_{k}}^{-1}$ be bounded, independently of $n,\left(\alpha_{0}, \alpha_{1}, \ldots, \alpha_{n-1}\right)$, and $k$.

We give a particular example of application, used in [6] and [5]. We refer to [6], and more generally to the book [15], for details about semisimple Lie groups and locally symmetric space of non-positive curvature. Let $G$ denote a non-compact connected simple Lie group with finite center. Let $K<G$ be a maximal compact subgroup, and $\mathbf{S}=G / K$ be the associated symmetric space. For a lattice $\Gamma<G$ we write $\mathbf{X}=\Gamma \backslash G$ and $\mathbf{Y}=\Gamma \backslash G / K$, the latter being a locally symmetric space of non-positive curvature.

On $T^{*} \mathbf{S}$, consider the algebra $\mathcal{H}$ of smooth $G$-invariant hamiltonians, that are polynomial in the fibers of the projection $T^{*} \mathbf{S} \longrightarrow \mathbf{S}$. The structure theory of semisim- 
ple Lie algebras shows that $\mathcal{H}$ is isomorphic to a polynomial ring in $r$ generators (where $r$ is the real rank of $G$ ). Moreover, the elements of $\mathcal{H}$ commute under the Poisson bracket. Thus, we have on $T^{*} \mathbf{S}$ a family of $r$ independent commuting Hamiltonians $H_{1}, \ldots, H_{r}$. Since the corresponding hamiltonian flows are $G$-equivariant, they descend to the quotient $T^{*} \mathbf{Y}$.

We apply the discussion above to a Hamiltonian $H \in \mathcal{H}$. The local dynamical properties of the flow $\left(\Phi_{H}^{t}\right)$, and in particular the invariant foliations, are best understood using the group-theoretical language.

We denote $\mathfrak{g}$ the Lie algebra of $G, \mathfrak{k}$ the Lie algebra of $K$, and $\mathfrak{a}$ a maximal abelian subalgebra of $\mathfrak{g}$, orthogonal to $\mathfrak{k}$ for the Killing form. The dimension of $\mathfrak{a}$ is the real rank $r$. For $\alpha$ in the dual $\mathfrak{a}^{*}$, let $\mathfrak{g}_{\alpha}=\{X \in \mathfrak{g}, \forall H \in \mathfrak{a}: \operatorname{ad}(H) X=\alpha(H) X\}$, and let $\Delta=\left\{\alpha \in \mathfrak{a}^{*} \backslash\{0\}, \mathfrak{g}_{\alpha} \neq\{0\}\right\}$ be the set of roots of $\mathfrak{g}$ with respect to $\mathfrak{a}$. For $\alpha \in \Delta$, we denote by $m_{\alpha}$ the dimension of $\mathfrak{g}_{\alpha}$. We have $\mathfrak{g}_{0}=\mathfrak{a} \oplus \mathfrak{m}$ where $\mathfrak{m}=Z_{\mathfrak{k}}(\mathfrak{a})$, the centralizer of $\mathfrak{a}$ in $\mathfrak{k}$. In $\Delta$, one can define a notion of positivity, and we will denote by $\Delta^{+}$the set of positive roots, by $\Delta^{-}=-\Delta^{+}$the set of negative roots. Writing $\mathfrak{n}=\oplus_{\alpha>0} \mathfrak{g}_{\alpha}$ and $\overline{\mathfrak{n}}=\Theta \mathfrak{n}=\oplus_{\alpha<0} \mathfrak{g}_{\alpha}$, we have $\mathfrak{g}=\mathfrak{n} \oplus \mathfrak{a} \oplus \mathfrak{m} \oplus \overline{\mathfrak{n}}$. Finally, let $N, A<G$ be the connected subgroups corresponding to the subalgebras $\mathfrak{n}, \mathfrak{a} \subset \mathfrak{g}$ respectively, and let $M=Z_{K}(\mathfrak{a})$.

We consider an open set $O \subset T^{*} \mathbf{Y}$ such that the differentials $\left(d H_{1}, \ldots, d H_{r}\right)$ are everywhere independent on $O$. It is known that any common energy layer $\left\{H_{1}=\right.$ $\left.E_{1}, \ldots, H_{r}=E_{r}\right\} \subset T^{*} \mathbf{Y}$, where the differentials $d H_{i}$ are independent, may naturally be identified (in a $G$-equivariant way) with $G / M[14]$. We thus have an equivariant map $O \longrightarrow \mathbb{R}^{r} \times G / M$ which is a diffeomorphism onto its image. In all that follows, we identify $O$ with an open subset of $\mathbb{R}^{r} \times G / M$. Under this identification, the action of $\Phi_{H}^{t}$ is transported to

$$
\left(E_{1}, \ldots, E_{r}, \rho M\right) \mapsto\left(E_{1}, \ldots, E_{r}, \rho e^{t a_{E_{1}}, \cdots, E_{r}} M\right),
$$

where $a_{E_{1}, \ldots, E_{r}} \in \mathfrak{a}$ depends smoothly on $E_{1}, \ldots, E_{r}$, and linearly on $H$ - see $[14,6]$ for detailed explanations. The foliation $\mathcal{F}$ can be described as follows : the leaf of $\left(E_{1}, \ldots, E_{r}, \rho M\right) \in \mathbb{R}^{r} \times G / M$ is $\left(E_{1}, \ldots, E_{r}\right) \times\{\rho a \bar{n} M, a \in A, \bar{n} \in \bar{N}\}$.

We assume that each $O_{k}$ is small enough so that, for any given $\left(E_{1}, \ldots, E_{r}, \rho M\right) \in$ $O_{k}$, the map

$$
\begin{aligned}
\mathbb{R}^{r} \times \mathfrak{n} \times \mathfrak{a} \times \overline{\mathfrak{n}} & \longrightarrow \mathbb{R}^{r} \times G / M \\
\left(\varepsilon_{1}, \ldots, \varepsilon_{r}, X, Y, Z\right) & \mapsto\left(\varepsilon_{1}, \ldots, \varepsilon_{r}, \rho e^{X} e^{Y} e^{Z} M\right)
\end{aligned}
$$

is a local diffeomorphism from a neighbourhood of $\left(E_{1}, \ldots, E_{r}, 0,0,0\right)$ onto $O_{k}$. In such coordinates, the leaves of the foliation $\mathcal{F}$ are then given by the equations $\left(\varepsilon_{1}, \ldots, \varepsilon_{r}, X\right)=$ cst.

Let $\left(\alpha_{0}, \ldots, \alpha_{n-1}\right)$ be such that $O_{\alpha_{0}} \cap \Phi_{H}^{-\tau}\left(O_{\alpha_{1}}\right) \cap \ldots \cap \Phi_{H}^{-(n-1) \tau}\left(O_{\alpha_{n-1}}\right) \neq \emptyset$. We can then take $\rho=\rho_{\alpha_{k}}$ and $\left(E_{1}, \ldots, E_{r}\right)$ such that $\left(E_{1}, \ldots, E_{r}, \rho_{\alpha_{k}} M\right) \in O_{\alpha_{k}}$ and $\left(E_{1}, \ldots, E_{r}, \rho_{\alpha_{k+1}} M\right)=\Phi_{H}^{\tau}\left(E_{1}, \ldots, E_{r}, \rho_{\alpha_{k}} M\right)$. As explained in the previous paragraph, fixing $\rho_{\alpha_{k}}$ allows to identify $O_{\alpha_{k}}$ with a subset of $\mathbb{R}^{r} \times \mathfrak{n} \times \mathfrak{a} \times \overline{\mathfrak{n}}$; and we denote $\left(\varepsilon_{1}, \ldots, \varepsilon_{r}, X, Y, Z\right)$ these coordinates.

Denote by $d$ the dimension of $\mathbf{S}$ (note that $d=r+\operatorname{dim} \mathfrak{n}=r+\sum_{\alpha \in \Delta^{+}} m_{\alpha}$ ). By the Darboux-Lie theorem [21], we can find some coordinate system $\Psi_{k}=$ $\left(x_{1}^{k}, \ldots, x_{d}^{k}, \xi_{1}^{k}, \ldots, \xi_{d}^{k}\right): O_{\alpha_{k}} \longrightarrow \mathbb{R}^{2 d}$ mapping $\omega$ to $\omega_{o}$, and such that $\left(\xi_{1}^{k}, \ldots, \xi_{d}^{k}\right)=$ $\left(\varepsilon_{1}, \cdots, \varepsilon_{r}, X\right)$. The canonical transformations $\kappa_{k}=\Psi_{k+1} \Phi_{H}^{\tau} \Psi_{k}^{-1}$ preserve the horizontal foliation of $\mathbb{R}^{2 d}$, hence they are of the form $\kappa_{k}:(x, \xi) \mapsto\left(x^{\prime}, \xi^{\prime}=p_{k}(\xi)\right)$. 
It turns out, in this particular case, that the maps $p_{k}$ are all the same, and of the particular form

$$
p_{k}\left(\varepsilon_{1}, \cdots, \varepsilon_{r}, X\right)=\left(\varepsilon_{1}, \ldots, \varepsilon_{r}, A d\left(e^{-\tau a_{\varepsilon_{1}}, \cdots, \varepsilon_{r}}\right) . X\right),
$$

where $a_{\varepsilon_{1}, \ldots, \varepsilon_{r}} \in \mathfrak{a}$ depends smoothly on $\varepsilon_{1}, \ldots, \varepsilon_{r}$. The linear maps $A d\left(e^{-\tau a_{\varepsilon_{1}, \ldots, \varepsilon_{r}}}\right)$ acting on $\mathfrak{n}$ are all simultaneously diagonalizable, the eigenspaces being the root spaces $\mathfrak{g}_{\alpha}$, with eigenvalue $e^{-\tau \alpha\left(a_{\varepsilon_{1}, \ldots, \varepsilon_{r}}\right)}$. We are thus in a case of application of Theorem 2.3 provided that condition (2.1) is satisfied; in our case this holds if and only if $\alpha\left(a_{\varepsilon_{1}, \ldots, \varepsilon_{r}}\right) \geq 0$ for all $\alpha \in \Delta^{+}$, since each $p_{k}$ is a linear map. This means that we want the leaves of $\mathcal{F}$ to be expanded under the action of $\kappa_{k}$. If we fix $J \subset \Delta^{+}$arbitrarily, the map $\kappa_{k}$ preserves the coisotropic foliation by the leaves $\left(\varepsilon_{1}, \ldots, \varepsilon_{r}, X_{J}\right)=c s t$ (where any $X \in \mathfrak{n}$ is decomposed into $X=\sum_{\alpha \in \Delta^{+}} X_{\alpha}, X_{\alpha} \in \mathfrak{g}_{\alpha}$, and $X_{J}$ is defined by $\left.X_{J}=\sum_{\alpha \in J} X_{\alpha}\right)$.

COROllary 5.1. Assume that $H$ and $O$ are such that $\alpha\left(a_{\varepsilon_{1}, \ldots, \varepsilon_{r}}\right) \geq 0$ for all $\left(\varepsilon_{1}, \ldots, \varepsilon_{r}, \rho M\right) \in O$ and all $\alpha \in \Delta^{+}$. Fix a subset $J \subset \Delta^{+}$of the sets of roots.

Fix $\mathcal{K}>0$ arbitrary. Then, for any $n \leq \mathcal{K}|\log h|$, and for every sequence $\left(\alpha_{0}, \ldots, \alpha_{n-1}\right)$,

$$
\left\|\prod_{k=0}^{n-1} \hat{\chi}_{\alpha_{k+1}} e^{-\frac{i \tau h \hat{H}}{h}} \hat{\chi}_{\alpha_{k}}\right\| \leq \sup _{\left(\varepsilon_{1}, \ldots, \varepsilon_{r}, \rho M\right) \in O} \prod_{\alpha \in \Delta^{+} \backslash J}\left(\frac{e^{-n \tau \alpha\left(a_{\varepsilon_{1}}, \ldots, \varepsilon_{r}\right)}}{2 \pi h}\right)^{m_{\alpha} / 2}
$$

for $h>0$ small enough.

In applications, one gets the best possible bound by choosing $J$ such that

$$
\frac{e^{-n \tau \alpha\left(a_{\varepsilon_{1}, \ldots, \varepsilon_{r}}\right)}}{2 \pi h}<1
$$

for all $\alpha \in \Delta^{+} \backslash J$. If $n$ is small, $J=\Delta^{+}$is the best possible choice and one gets the trivial bound $\left\|\prod_{k=0}^{n-1} \hat{\chi}_{\alpha_{k+1}} e^{-\frac{i \tau h \hat{H}}{h}} \hat{\chi}_{\alpha_{k}}\right\| \leq 1$. Taking $J \neq \Delta^{+}$starts to be interesting for $n=\mathcal{K}|\log h|$ with $\mathcal{K}$ large enough.

REMARK 5.2. In the situation of [6], we actually do not have $\alpha\left(a_{\varepsilon_{1}, \ldots, \varepsilon_{r}}\right) \geq 0$, but $\alpha\left(a_{\varepsilon_{1}, \ldots, \varepsilon_{r}}\right) \geq-\delta$, where $\delta>0$ can be made arbitrarily small by conveniently choosing the set $O$. Once $\mathcal{K}$ is given, we can choose $\delta$ small enough (and $O$ ) so that the proof of Section 4.2 works for $n=\mathcal{K}|\log h|$.

In the special case $G=S O_{o}(d, 1), \mathbf{Y}$ is a hyperbolic manifold of dimension $d$. We have $r=1$, and $\mathcal{H}$ is generated by the laplacian $\triangle$. We take $\hat{H}=-\frac{h^{2} \triangle}{2}$, $J=\emptyset$, and assume that the pseudodifferential operators $\hat{\chi}_{k}$ are all microsupported in $\left\{(x, \xi) \in T^{*} \mathbf{Y},\|\xi\| \in[1-\eta, 1+\eta]\right\}$ for some small $\eta>0$. With the previous notation, this implies that $\alpha\left(a_{\varepsilon_{1}, \ldots, \varepsilon_{r}}\right)$ is in $[1-\eta, 1+\eta]$. We thus obtain the estimate

$$
\left\|\prod_{k=0}^{n-1} \hat{\chi}_{\alpha_{k+1}} e^{-\frac{i \tau h \hat{H}}{h}} \hat{\chi}_{\alpha_{k}}\right\| \leq\left(\frac{e^{-n \tau(1-\eta)}}{2 \pi h}\right)^{\frac{d-1}{2}}
$$

If $n \leq \frac{|\log h|}{1-\eta}$, this bound is trivial and it is preferrable to take $J=\Delta^{+}$as we said above. But as soon as $n \geq \frac{|\log h|}{1-\eta}$, the bound is optimized by taking $J=\emptyset$. 
We note that the result proved in $[1,3]$, which was only based on the idea of Theorem 2.2, was

$$
\left\|\prod_{k=0}^{n-1} \hat{\chi}_{\alpha_{k+1}} e^{-\frac{i \tau h \hat{H}}{h}} \hat{\chi}_{\alpha_{k}}\right\| \leq \frac{1}{(2 \pi h)^{d / 2}} e^{-n \tau \frac{(d-1)}{2}(1-\eta) .}
$$

We see that Theorem 2.3 allows to improve the prefactor $\frac{1}{(2 \pi h)^{d / 2}}$ to $\frac{1}{(2 \pi h)^{(d-1) / 2}}$, as needed in [5].

REMARK 5.3. Versions of the hyperbolic dispersion estimate have also been proved for more general uniformly hyperbolic dynamical systems [1, 3, 17, 19], and even for certain non-uniformly hyperbolic systems [20]. We refer the reader to [16] for an expository paper. It is not clear to me whether the new presentation (and improvement) introduced here can be used for those systems. Indeed, there is in general no smooth lagrangian foliations preserved by the hamiltonian flow, and so one cannot hope that the symplectic changes of coordinates $\Psi_{k}$ used above will have uniformly bounded derivatives. Control of high order derivatives is crucial when one applies the techniques of semiclassical analysis (method of stationary phase, integration by parts,...) It is a drawback of semiclassical analysis that it cannot deal with symplectic transformations of low regularity: I don't know if this obstacle can be overcome.

\section{REFERENCES}

[1] N. Anantharaman, Entropy and the localization of eigenfunctions, Ann. of Math. (2), 168:2 (2008), pp. 435-475.

[2] N. Anantharaman, A hyperbolic dispersion estimate, with applications to the linear Schrödinger equation, to appear in Proceedings of the ICM 2010.

[3] N. Anantharaman and S. Nonnenmacher, Half-delocalization of eigenfunctions for the laplacian on an Anosov manifold, Festival Yves Colin de Verdière. Ann. Inst. Fourier (Grenoble), $57: 7$ (2007), pp. 2465-2523.

[4] N. Anantharaman, H. Koch, and S. Nonnenmacher, Entropy of eigenfunctions, New Trends of Mathematical Physics, selected contributions of the 15th International Congress on Mathematical Physics, Springer (2009), pp. 1-22.

[5] N. Anantharaman and G. Rivière, Dispersion and controllability for the Schrödinger equation on negatively curved manifolds, arXiv:1007.4343, to appear in Analysis \& PDE.

[6] N. Anantharaman and L. Silberman, Asymptotic distribution of eigenfunctions on locally symmetric spaces, arXiv:1009.4927.

[7] N. Burq, C. Guillarmou, and A. Hassell, Strichartz estimates without loss on manifolds with hyperbolic trapped geodesics, preprint, arXiv:0907.3545.

[8] H. Christianson, Semiclassical non-concentration near hyperbolic orbits, J. Funct. Anal., 262 (2007), pp. 145-195; ibid, Dispersive estimates for manifolds with one trapped orbit, Comm. PDE, 33 (2008), pp. 1147-1174.

[9] H. Christianson, Cutoff resolvent estimates and the semilinear Schrödinger equation, Proc. AMS, 136 (2008), pp. 3513-3520.

[10] H. Christianson, Applications to cut-off resolvent estimates to the wave equation, Math. Res. Lett., 16:4 (2009), pp. 577-590.

[11] K. DATCHev, Local smoothing for scattering manifolds with hyperbolic trapped sets, Comm. Math. Phys., 286, no. 3, pp. 837-850.

[12] S.-I. DoI, Smoothing effects of Schrödinger evolution groups on Riemannian manifolds, Duke Math. J., 82 (1996), pp. 679-706.

[13] L. C. Evans AND M. ZWORSki, Lectures on semiclassical analysis (version 0.95), avalaible at http://math.berkeley.edu/ zworski/semiclassical.pdf, (2011).

[14] J. HilgerT, An ergodic Arnold-Liouville theorem for locally symmetric spaces, Twenty years of Bialowieza: a mathematical anthology, pp. 163-184, World Sci. Monogr. Ser. Math., 8, World Sci. Publ., Hackensack, NJ, 2005.

[15] A. Knapp, Lie groups beyond an introduction, Progress in Mathematics, 140. Birkhäuser Boston, Inc., Boston, MA, 1996. 
[16] S. Nonnenmacher, Entropy of chaotic eigenstates, Notes of the minicourse given at the workshop "Spectrum and dynamics", Centre de Recherches Mathématiques, Montréal, April 2008, arXiv:1004.4964.

[17] S. Nonnenmacher And M. Zworski, Quantum decay rates in chaotic scattering, Acta Mathematica, 203:2 (2009), pp. 149-233.

[18] S. Nonnenmacher And M. Zworski, Semiclassical resolvent estimates in chaotic scattering, Appl. Math. Res. Express, (2009), pp. 74-86.

[19] G. Rivière, Entropy of semiclassical measures in dimension 2, Duke Math. J., 155:2 (2010), pp. 271-335.

[20] G. RivièRE, Entropy of semiclassical measures for nonpositively curved surfaces, Annales Henri Poincaré, 11:6 (2010), pp. 1085-1116.

[21] I. Vaisman, Basics of Lagrangian foliations, Publ. Mat., 33:3 (1989), pp. 559-575. 
